\title{
openheart Guideline-defined futility or patient- reported outcomes to assess treatment success after TAVI: what to use? Results from a prospective cohort study with long-term follow-up
}

\author{
Martijn Stefan van Mourik, Jeroen Vendrik, Mohammad Abdelghani, \\ Floortje van Kesteren, Jose P S Henriques, Antoine H G Driessen, \\ Joanna J Wykrzykowska, Robbert J de Winter, Jan J Piek, Jan G Tijssen, \\ Karel T Koch, Jan Baan, M Marije Vis
}

\begin{abstract}
- Additional material is published online only. To view please visit the journal online (http://dx.doi.org/10.1136/ openhrt-2018-000879).

To cite: van Mourik MS, Vendrik J, Abdelghani M, et al. Guideline-defined futility or patient-reported outcomes to assess treatment success after TAVI: what to use? Results from a prospective cohort study with long-term follow-up. Open Heart 2018;5:e000879. doi:10.1136/ openhrt-2018-000879
\end{abstract}

MSvM and JV contributed equally.

Received 11 June 2018 Revised 27 July 2018 Accepted 14 August 2018

Check for updates

(c) Author(s) (or their employer(s)) 2018. Re-use permitted under CC BY-NC. No commercial re-use. See rights and permissions. Published by BMJ.

Heart Center, Department of Cardiology, Amsterdam Cardiovascular Sciences, Amsterdam UMC, University of Amsterdam, Amsterdam, The Netherlands

Correspondence to DrM Marije Vis; m.m.vis@ amc.nl

\section{ABSTRACT}

Objective Transcatheter aortic valve implantation (TAVI) provides a significant symptom relief and mortality reduction in most patients; however, a substantial group of patients does not experience the same beneficial results according to physiciandetermined outcomes.

Methods Single-centre prospective design; the population comprises all consecutive patients undergoing TAVI in 2012-2017. TAVI futility was defined as the combined endpoint of either no symptomatic improvement or mortality at 1 year. We actively gathered telephone followup using a predefined questionnaire.

Results Guideline defined TAVI futility was present in 212/741 patients. Multivariate regression showed lower albumin and non-transfemoral approach to be predictive for futility. In addition to these, chronic obstructive pulmonary disease, lower estimated glomerular filtration rate, atrial fibrillation, low-flowlow-gradient aortic stenosis and lower Body Mass Index were predictive for 1-year mortality. Patients who showed symptomatic benefit estimated the percentage in which their symptoms were remedied higher than patients who did not $(80 \%$ vs $60 \%, p<0.001)$. Guideline-defined TAVI futility occurs frequently, contrasting with patient-reported outcome measures (PROMs). The vast majority in both groups would again choose for TAVI treatment.

Conclusion Lower albumin and non-transfemoral access route were predictors for guideline-defined TAVI futility, defined as mortality within 1 year or no objective symptomatic improvement in New York Heart Association class. Futility according to this definition occurred frequently in this study, contrasting with much more positive PROMs. The majority of patients would undergo a TAVI again, underlining the patients' experienced value of TAVI and putting the definition of TAVI futility further on debate. In the near future, lessstrict criteria for TAVI futility, that is, using a shorter warranted life expectancy and incorporating patients' perceived outcomes, should be used.

\section{Key questions}

What is already known about this subject?

- Despite the fact that transcatheter aortic valve implantation (TAVI) provides a significant symptom relief and mortality reduction in most patients, a substantial group of patients does not experience the same beneficial results or dies shortly after TAVI. Multiple studies derived from large registries identified several predictors for poor outcome.

What does this study add?

- In this study, we assessed whether these results also apply for a real-world clinical care setting using our prospective monocentre registry derived from regular clinical care and supplemented with patient-reported outcomes.

How might this impact on clinical practice?

- This study sheds new light on the actual, and the patient-experienced, effects of TAVI treatment and further elucidates the baseline characteristics predicting futile TAVI according to the current guidelines. These predicting factors could be used to inform each specific patient on his or her prognosed benefit-risk and benefit-cost trade-off in order to improve shared decision-making and manage the patient's expectations.

\section{INTRODUCTION}

Transcatheter aortic valve implantation (TAVI) has evolved into an established treatment for patients with severe aortic valve stenosis at intermediate, high or prohibitive risk for surgical aortic valve replacement. In elderly patients at increased surgical risk, TAVI is superior in terms of mortality to medical therapy in extreme-risk patients, non-inferior or superior to surgery in highrisk patients and non-inferior to surgery, 
and even superior when transfemoral access is possible in intermediate-risk patients. Finally, an estimated life expectancy of at least 1 year is warranted in the recently used guidelines. ${ }^{12}$ Surgical risk models currently used in TAVI practice poorly predict TAVI outcomes ${ }^{34}$; however, specific risk stratification models are just recently developed and not commonly used in clinical practice. Further broadening of the indication for TAVI to lowerrisk patients increases the need for proper patient risk stratification and outcome prediction.

Despite the fact that TAVI provides a significant symptomatic improvement and mortality reduction in most patients, a substantial group of patients does not experience the same beneficial results or dies shortly after receiving TAVI. Recent randomised trials report 1-year mortality rates varying from $6.7 \%$ to $14.5 \%,{ }^{5} 6$ even in intermediate-risk patients, indicating possibly that these patients were better off when treated otherwise or not at all.

Current data indicate that TAVI has a high likelihood to be futile or result in a poor outcome (ie, not yielding a positive functional result or survival benefit during 1-year follow-up ${ }^{78}$ ) in patients with severe pulmonary disease, ${ }^{9} 10$ severe renal dysfunction, ${ }^{11}{ }^{12}$ low-flow-low-gradient aortic stenosis (LF-LG AS), pulmonary hypertension or severe mitral regurgitation. ${ }^{7}$ Multiple studies derived from large registries (PARTNER, CoreValve, FRANCE-2) identified several predictors for poor outcome. ${ }^{13-15}$ In this study, we assessed whether these results also apply for a realworld clinical care setting using our prospective mono centre registry derived from regular clinical care and supplemented with patient-reported outcome measures (PROMs).

Modern 'value-based' healthcare focuses on optimising the benefit-risk and the benefit-cost trade-offs as well as on shared decision-making, that is, involving the patient to make his own informed choice. ${ }^{16}$ The procedural risks involved with TAVI, although by definition lower than in surgical valve replacement, are still not negligible, as are the costs. Appropriate patients' selection and foreseeing their use/futility likelihood are, thus, important to manage patient expectations and to best use the limited healthcare resources.

The aim of this study was twofold: (1) to explore predictors of symptomatic improvement, 1-year mortality and the combined endpoint of guideline-defined TAVI futility and (2) to assess the subjective patient-expressed satisfaction after TAVI (using the PROMs).

\section{METHODS}

\section{Patient selection and data acquisition}

The population comprised all consecutive patients with symptomatic aortic valve stenosis who underwent TAVI between January 2012 and January 2017 in the Academic Medical Center (AMC), Amsterdam, The Netherlands. The Ethics Committee of the AMC approved this research with a waiver. All data were entered into a dedicated prospective TAVI registry with an active follow-up of clinical and patient-reported outcomes.

The decision for TAVI was made by a dedicated multidisciplinary TAVI team. The transfemoral approach was the default access option. In patients unsuitable for transfemoral approach, the direct aortic or transapical approach was used. Device sizing was based on multislice CT measurements of the annulus size. Both the EuroSCORE II and the Society of Thoracic Surgeons Predicted Risk of Mortality (STS-PROM, calculated with the Online STS Adult Cardiac Surgery Risk Calculator V.2.81) were used for operative mortality risk stratification. All used definitions are in accordance with the most recent guidelines. $^{1217}$

\section{Outcomes and definitions}

Primary outcomes were (1) 1-year mortality and (2) New York Heart Association (NYHA) functional class improvement. The Valve Academic Research Consortium 2 criteria were used for outcome definitions. ${ }^{17}$ Mortality data were obtained from the Dutch national municipal register on 30 April 2017, ensuring complete follow-up.

In August 2017, all patients who were alive were contacted by telephone. Patients were asked a predefined set of questions, including complications after hospital discharge, current symptoms and the patient-perceived treatment effect (online supplement S1). When patients could not be reached, the first contact person or general practitioner was contacted for follow-up information retrieval. Patients who were not reached after trying at least five times were marked as lost to follow-up (table 1 and figure 1).

Difference in NYHA functional class from baseline to at least 30 days' follow-up was assessed. Patients who had NYHA class 1 , that is, no exertional dyspnoea, and were treated for their symptomatic AS because of angina pectoris, syncope or extreme fatigue were excluded from this analysis. Patients who either had no 30-60 day follow-up of functional status (NYHA) or died before the 60-day time point were also excluded from this analysis (table 2 and figure 2). The presence of a LF-LG AS was defined as an aortic valve area (AVA) $<1 \mathrm{~cm}^{2}$ or indexed AVA $<0.6 \mathrm{~cm}^{2} / \mathrm{m}^{2}$, a mean AV gradient $<40 \mathrm{~mm} \mathrm{Hg}$ and a left ventricular ejection fraction $<50 \%$, according to most recent guidelines. ${ }^{12} 18$ Albumin cut-off of $40 \mathrm{~g} / \mathrm{L}$ was based on the lowest quartile of the total population.

TAVI was labelled futile if there was no improvement of NYHA functional class or the patient dies within 1 year after the procedure. Residual functional impairment was defined as having a NYHA class greater than 1 after TAVI.

\section{Statistical analysis}

Categorical variables are presented as numbers with percentages and compared between the groups with Fisher's exact test. For continuous data, normality was checked, and data are presented as means with SD or medians with IQRs and compared using an unpaired Student's t-test or Mann-Whitney U test, as appropriate. 
Cumulative survival was estimated using Kaplan-Meier analysis and compared between groups using log-rank test and Cox proportional hazards models. Univariate and multivariate logistic regression was performed to identify potential predictors of TAVI futility, residual functional impairment and 1-year mortality. All variables with a $\mathrm{p}$ value $<0.10$ in the univariate model were entered in the multivariable analysis. For all analyses, a $\mathrm{p}$ value $<0.05$ was considered statistically significant. Analyses were performed in SPSS V.24.0 (IBM) and R (V.3.3.3; R Foundation for Statistical Computing, Vienna, Austria).

\section{RESULTS}

\section{Baseline characteristics}

The total study population consisted of 809 patients (figure 1). The median age was 80 years and $45 \%$ were men (table 2). Mean AVA was $0.82 \pm 0.27 \mathrm{~cm}^{2}$ and mean AV gradient was $65 \pm 23 \mathrm{~mm} \mathrm{Hg}$. Predicted surgical mortality risk was for the STS-PROM score $5.46 \pm 4.63 \%$ and for the EuroSCORE II, $5.71 \pm 4.90 \%$. The vast majority of the

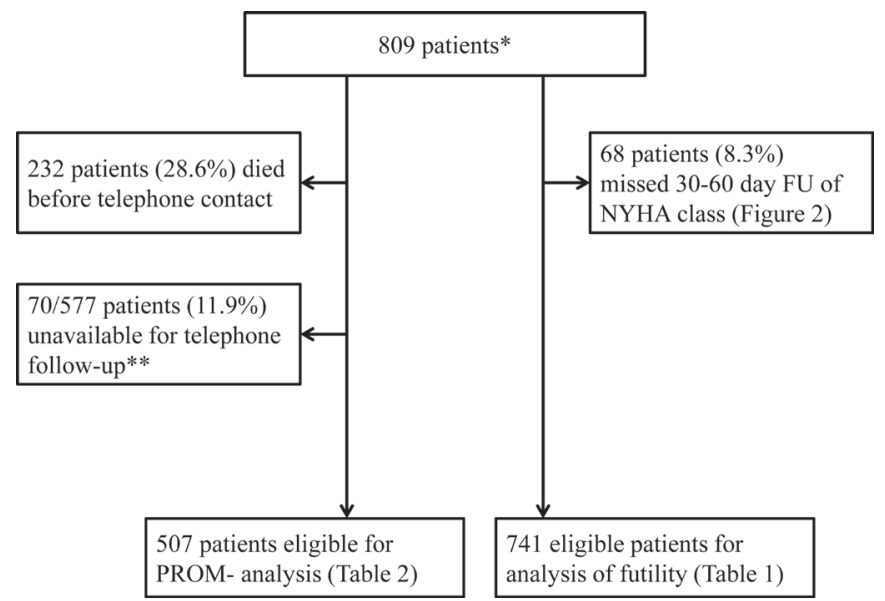

Figure 1 Flowchart of study and patient selection. *From January 2012 to December 2016. **Either not reached after at least five tries or not able to give adequate answers by telephone due to deafness or dementia. FU, follow-up; NYHA, New York Heart Association; PROM, patient-reported outcome measure. patients $(\mathrm{n}=779,96.2 \%)$ had complaints of dyspnoea prior to TAVI (figure 2). Complete follow-up of NYHA class and PROMs was retrieved in 741 and 507 patients, respectively.

\section{One-year mortality}

One-year mortality was compared between the subgroups of patients, based on the presence of different baseline and procedural characteristics (figure 3). Lower survival was seen in patients with atrial fibrillation $(90.3 \%$ vs $80.0 \%, \mathrm{p}<0.0001)$, chronic obstructive pulmonary disease (COPD) $(87.8 \%$ vs $81.2 \%, \mathrm{p}=0.0092)$, LF-LG AS $(88.9 \%$ vs $77.3 \%, \mathrm{p}<0.0001)$ and in patients with impaired left ventricular function $(75.9 \%$ vs $82.4 \%$ vs $90.3 \%$, for moderate/severe impaired, mild impaired and good left ventricular function, respectively, $\mathrm{p}<0.0001)$. Patients without residual impairment (NYHA class 1 after TAVI) had a significant better 1-year survival than the patients with residual impairment (NYHA class $>1$ ). Patients with symptomatic improvement on NYHA class also showed a better 1-year survival; however, this was statistically non-significant $(92.9 \%$ vs $89.0 \%, \mathrm{p}=0.14)$.

Multivariable regression revealed that the presence of atrial fibrillation (AF) (OR 2.06 (1.35-3.19), p<0.001), lower eGFR (OR 0.99 (0.98-1.00), $\mathrm{p}=0.035)$, lower baseline albumin levels (OR $0.94(0.89-0.99)$ per point $\mathrm{g} / \mathrm{L}, \mathrm{p}=0.022$ ), lower Body Mass Index (BMI) (OR 0.93 (0.89-0.98) per point $\left.\mathrm{kg} / \mathrm{m}^{2}, \mathrm{p}=0.005\right)$, LF-LG AS (OR 1.84 (1.17-2.89), $\mathrm{p}=0.008)$ and non-transfemoral access route (OR $0.60(0.39-0.95), \mathrm{p}=0.019)$ were independent predictors for 1-year mortality (online supplement S3).

\section{Symptomatic improvement and residual impairment}

The distribution of NYHA class before and after TAVI and improvement in functional status is depicted in figure 2. Benefit of TAVI in symptomatic status (NYHA class decrease) was seen in $568(83.7 \%)$ patients; no result or worsening of the symptomatic status (no NYHA class decrease) was seen in $173(16.3 \%)$ patients. Residual impairment was seen in 293/741 $(39.5 \%)$ patients. Multivariate logistic regression revealed no factors as independent predictors for an improvement in NYHA class. 
Table 2 Baseline characteristics of the whole cohort and compared between the designated futile TAVI group and control group

\begin{tabular}{lcccc}
\hline & All patients & Controls & Futile TAVI* & P values† $^{*}$ \\
\hline $\mathrm{n}$ & 741 & 529 & 212 & \\
\hline Age (years, median (IQR)) & $81.9(77.3-85.3)$ & $82.1(77.4-85.3)$ & $81.5(77.3-85.3)$ & 0.459 \\
\hline BMI (kg/m², mean (SD)) & $27.7(5.08)$ & $27.79(5.02)$ & $27.49(5.23)$ & 0.469 \\
\hline Male gender (n (\%)) & $326(44.0)$ & $229(43.3)$ & $97(45.8)$ & 0.597 \\
\hline STS-PROM (mean (SD)) & $5.49(4.73)$ & $5.23(4.78)$ & $6.15(4.55)$ & 0.017 \\
\hline EuroSCORE II (mean (SD)) & $5.75(4.93)$ & $5.40(4.29)$ & $6.64(6.18)$ & 0.002 \\
\hline Atrial fibrillation (n (\%)) & $316(42.6)$ & $214(40.5)$ & $102(48.1)$ & 0.068 \\
\hline COPD (n (\%)) & $231(31.2)$ & $149(28.2)$ & $82(38.7)$ & 0.007 \\
\hline COPD GOLD classification (mean (SD)) & $1.86(1.53)$ & $1.76(1.47)$ & $2.10(1.65)$ & 0.010 \\
\hline Diabetes mellitus (n (\%)) & $229(30.9)$ & $155(29.3)$ & $74(34.9)$ & 0.160 \\
\hline Current smoker (n (\%)) & $65(8.9)$ & $45(8.6)$ & $20(9.7)$ & 0.741 \\
\hline Previous stroke (n (\%)) & $80(10.8)$ & $61(11.5)$ & $19(9.0)$ & 0.375 \\
\hline Previous PCI (n (\%)) & $200(27.0)$ & $142(26.8)$ & $58(27.4)$ & 0.959 \\
\hline Previous CABG (n (\%)) & $100(13.5)$ & $70(13.2)$ & $30(14.2)$ & 0.832 \\
\hline Previous PM (n (\%)) & $77(10.4)$ & $55(10.4)$ & $22(10.4)$ & 1.000 \\
\hline Serum creatinine (mmol/L, mean (SD)) & $106.58(73.10)$ & $101.72(66.69)$ & $118.73(86.04)$ & 0.004 \\
\hline eGFR (mL/min/1.73 m², CKD-EPI, median (IQR)) & $53.81(37.36-72.32)$ & $55.29(39.29-75.22)$ & $48.91(31.36-69.48)$ & 0.003 \\
\hline Haemoglobin (mmol/L, median (IQR)) & $7.80(7.10,8.40)$ & $7.80(7.10,8.40)$ & $7.80(7.10,8.50)$ & 0.934 \\
\hline Albumin (g/L, median (IQR)) & $42(40-44)$ & $42(40-44)$ & $41(38-43)$ & $<0.001$ \\
\hline Serum NTproBNP (ng/L, median (IQR)) & $1603(693-3844)$ & $1462(648-3700)$ & $1891(812-4326)$ & 0.084 \\
\hline Aortic valve area (cm², mean (SD)) & $0.82(0.28)$ & $0.82(0.23)$ & $0.83(0.37)$ & 0.855 \\
\hline Aortic valve peak gradient (mm Hg, mean (SD)) & $65.1(22.3)$ & $65.7(21.7)$ & $63.6(23.5)$ & 0.250 \\
\hline Moderate to severe RV failure (n (\%)) & $72(9.7)$ & $46(8.7 \%)$ & $26(12.3 \%)$ & 0.181 \\
\hline SPAP over 60 mm Hg (n (\%)) & $252(34.1)$ & $190(36.0)$ & $62(29.2)$ & 0.096 \\
\hline Transfemoral access route (n (\%)) & $557(75.2)$ & $416(78.6)$ & $141(66.5)$ & 0.001 \\
\hline
\end{tabular}

${ }^{*}$ Guideline defined futile result=composite endpoint; either no decrease on NYHA class after 30-60 day follow-up or subject did not survive 1 year after procedure.

†P value for the comparison of designated futile TAVI versus the control group.

BMI, Body Mass Index; CABG, coronary artery bypass grafting; COPD, chronic obstructive pulmonary disease; eGFR, estimated glomerular filtration rate; GOLD, Global Initiative for Chronic Obstructive Lung Disease; NTproBNP, N-terminal prohormone brain natriuretic peptide; NYHA, New York Heart Association; PCI, percutaneous coronary intervention; PM, pacemaker; RV, right ventricle; SPAP, systolic pressure in arteria pulmonalis; STS-PROM, Society of Thoracic Surgeons Predicted Risk of Mortality; TAVI, transcatheter aortic valve implantation.

However, the presence of COPD (OR $1.90(1.35-2.67$, $\mathrm{p}<0.001)$, female gender (OR male gender; $0.62(0.45-$ $0.85), \mathrm{p}=0.003)$, lower albumin (OR $0.95(0.91-0.99)$ per point $\mathrm{g} / \mathrm{L}, \mathrm{p}=0.024)$ and eGFR (OR $0.99(0.99-1.00$ per point $\left.\mathrm{mL} / \mathrm{min} / 1.73 \mathrm{~m}^{2}, \mathrm{p}=0.050\right)$ levels were found as predicting factors for residual impairment.

\section{Futility}

The entire cohort was divided into two subgroups; $212 / 741$ patients $(28.7 \%)$ in the guideline defined futile group, of which 113 patients $(53.3 \%)$ died within 1 year after TAVI and 99 patients $(46.7 \%)$ showed no symptomatic improvement. The control group consisted of the remaining 529/741 patients $(71.3 \%)$. Estimated operative mortality risk was significantly higher in the futile group (STS-PROM $6.15 \pm 4.78 \%$ vs $5.23 \pm 4.78 \%, \mathrm{p}=0.017$ and EuroSCORE II; $6.64 \pm 6.18 \%$ vs $5.40 \pm 4.29 \%$, $\mathrm{p}=0.002$ ).
Patients in the futile group more frequently had a history of COPD (38.7\% vs $28.2 \%, \mathrm{p}=0.007)$ and a higher Global Initiative for Chronic Obstructive Lung Disease classification (2.10 vs $1.76, p=0.01$ ), indicating more severe COPD. Patients in the futile group had a higher mean serum creatinine $(118 \pm 86 \mathrm{mmol} / \mathrm{L}(1.33 \pm 0.97 \mathrm{mg} / \mathrm{dL})$ vs $102 \pm 67 \mathrm{mmol} / \mathrm{L}(1.15 \pm 0.76 \mathrm{mg} / \mathrm{dL}), \mathrm{p}=0.004)$ and a lower median eGFR (49 (31-69) $\mathrm{mL} / \mathrm{min}$ vs $55(39-75)$ $\mathrm{mL} / \mathrm{min}, \mathrm{p}=0.003$ ) (table 2).

Univariate logistic regression analysis showed COPD and eGFR to increase the odds for futile TAVI, and higher serum albumin and transfemoral access route lowering the odds for futile TAVI. After multivariable logistic regression analysis, the remaining statistically significant variables of futility were serum albumin (OR $0.93(0.89-0.97), \mathrm{p}<0.001)$ and femoral access route (OR 


\section{NYHA before and 30-60 days after TAVI}

NYHA 1 NYHA 2 NYHA 3 NYHA 4 Died within 60 days $=$ Missing

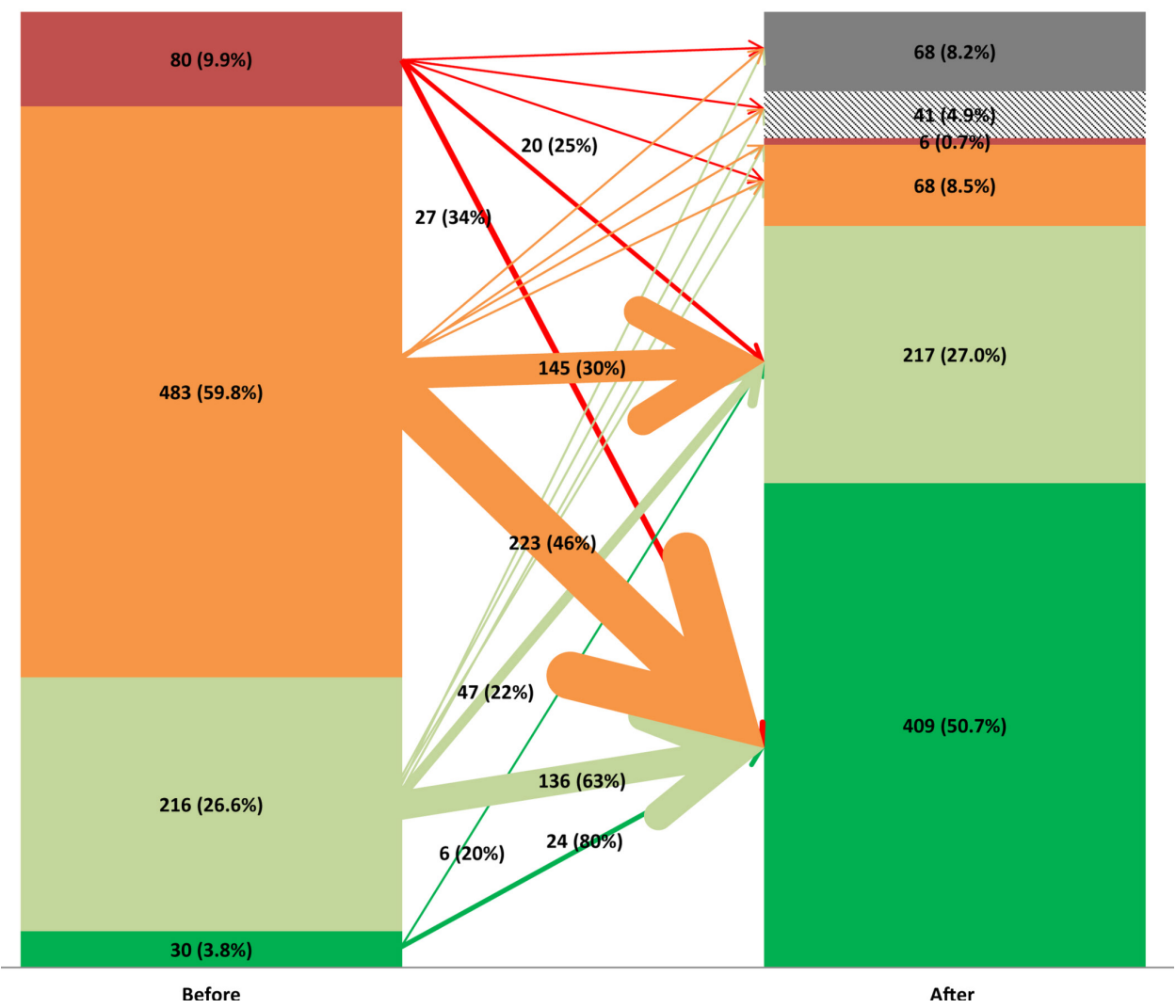

Figure 2 NYHA before and after at least 30-60 days after TAVI. The arrows depict the absolute number of patients going to (another) NYHA class after 30-60 day follow-up. The bigger the arrow, the larger the absolute number of patients. The two biggest subgroups out of each of the NYHA class before TAVI are also accompanied by a percentage, depicting the proportion of the total group moving to another NYHA class after TAVI. Percentages do not add up to 100 because of rounding errors. NYHA, New York Heart Association; TAVI, transcatheter aortic valve implantation.

$0.53(0.37-0.76), \mathrm{p}<0.001)$. Thus, lower levels of albumin and non-transfemoral access route increased the risk for futile TAVI. Lower eGFR and presence of COPD showed an insignificant trend for TAVI futility (online supplement S2).

\section{Patient-reported outcome measures}

A total of 507 patients were reached by telephone $(88.1 \%$ of eligible, alive patients at that moment), after a median follow-up of 757 days (IQR 465-1139). Baseline characteristics and NYHA class 30-60 days post-TAVI did not differ between reached and non-reached patients.

Patients with symptomatic improvement (based on NYHA class, $n=408,80.5 \%$ ) estimated their percentage in which their symptoms were remedied as $80 \%$, compared with $60 \%$ in the patients without any objective symptomatic improvement $(\mathrm{p}<0.001)$. The majority of patients $(68.6 \%)$ experienced a $>50 \%$ remedy of their main symptom, without significant difference between the groups with and without symptomatic improvement. Moreover, $90.6 \%$ of the symptomatically improved patients said they would undergo the procedure again, compared with $86.2 \%$ of the patients without symptomatic improvement $(\mathrm{p}=0.375)$.

\section{DISCUSSION}

In this study, we show the results of a single-centre TAVI cohort. This study sheds new light on the actual, and the patient-experienced, effects of TAVI treatment and further elucidates the baseline characteristics predicting futile TAVI. We found that the majority of patients have an improvement in NYHA class, in contrast to the fact that a large proportion still has residual impairment. We reported a discrepancy between the measured benefits and the perceived benefits: the number of patients who benefited from the TAVI procedure based on dyspnoea improvement was much lower than the number of patients who reported to have benefited. We added patient-reported outcomes, actively collected by telephone questionnaires, a measure which is often lacking in comparable studies. We believe that specifically in a frail and very elderly population, patient-reported outcomes are an important measure since the main focus of treatment is not solelyon life extension but the more on the patient-perceived experience in their remaining life span.

\section{Predicting futile TAVI}

Our aim was to find clinically useful predictors and not to create a complete model to predict futility. We found 

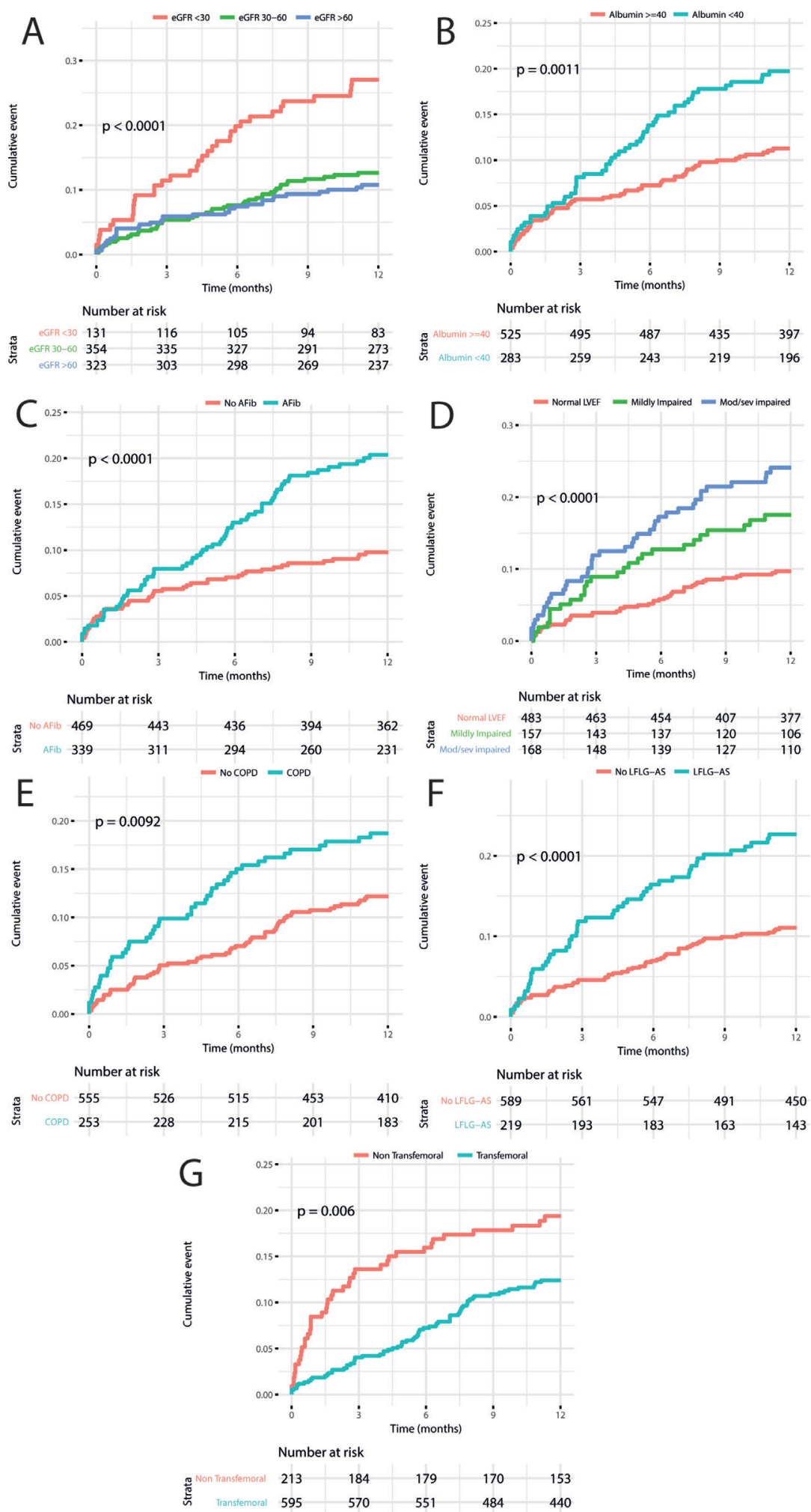

Figure $3 \mathrm{KM}$ analysis of 1-year mortality in the study population stratified according to different baseline characteristics. (A) Estimated glomerular filtration rate (eGFR, $\mathrm{mL} / \mathrm{min} / 1.73 \mathrm{~m}^{2}$ ). Pairwise log-rank testing using Benjamini-Hochberg correction for multiple testing showed a significant difference between the group with eGFR $<30$ and eGFR $30-60(p=0.00016)$ and eGFR $>60$ $(p<0.0001)$; however, there was no significant difference between the group eGFR $>60$ and eGFR $30-60$. (B) Serum albumin. (C) Presence of atrial fibrillation (Afib). (D) Left ventricular failure (LVEF, left ventricular ejection fraction). Pairwise log-rank testing using Benjamini-Hochberg correction for multiple testing showed a significant difference between the group with normal LVEF and mildly impaired LVEF $(p=0.011)$ and moderate/severe impaired $(p<0.0001)$; however, there was no significant difference between the group of mildly impaired LVEF and moderate/severe impaired LVEF ( $p=0.154)$. ( $E$ ) Presence of chronic obstructive pulmonary disease (COPD). (F) Presence of low-flow-low-gradient aortic stenosis (LF-LG AS). (G) Access route; transfemoral versus non-transfemoral. 
several predictors for our combined endpoint declaring futile TAVI. Lower albumin levels were likely corresponding to a poor nutritional status. Non-transfemoral access route was a strong predictor for TAVI futility, as earlier described. ${ }^{19}$ However, these results could have been biased by the fact that the non-transfemoral route was only used in patients unsuitable for transfemoral approach, corresponding with other comorbidities which may not always have been adjusted for. Furthermore, this is a combination between patient and device characteristics, of which the latter changed over time caused by technological developments. However, it is still important to incorporate possible access routes in clinical decision-making because it strongly predicts outcome.

When divided into two separate endpoints, respectively 1-year mortality and the absence of functional improvement, even more predictors were found. The presence of AF, LF-LG AS, COPD, declined renal function, lowered serum albumin and lower BMI were all significant predictors for 1-year mortality. These results can certainly be used to further clarify the expected harm and benefit for the patient before TAVI treatment.

In this study, no significant predictors for symptomatic improvement (in NYHA class) were found, which may be explained by the small amount of stepwise differentiation in the coarse NYHA scale. Significant predictors were found for the more crass endpoint of residual impairment. Residual impairment of functional capacity is common after TAVI as recently described in a Brazilian cohort. ${ }^{20}$ Residual impairment was described to be independently associated with an increased mid-term mortality, which we withal confirm judging from the KM curves we show (figure 3). We found that female gender, the presence of COPD, higher haemoglobin levels, lower BMI, declined renal function, lower albumin levels and the absence of diabetes mellitus were predicting factors for residual impairment. These predicting factors could be used to inform each specific patient on his or her prognosed benefit-risk and benefit-cost trade-off, in order to improve shared decision-making and better manage the patient' expectations.

\section{Futile TAVI?}

Guideline-defined futile TAVI occurred frequently in this study $(212 / 741 ; 28.6 \%)$, but comparable with other large cohort results. ${ }^{13}$ This is in part due to the fact that strict criteria were used based on the current guidelines and measures of symptomatic status by NYHA classification. Since the overall survival is very acceptable and the patients' opinion about the TAVI after the treatment is mostly positive, these futility criteria could be too strict. Other definitions also apply for this patient population and are evolving over time. Earlier studies describe pre-procedural diuretic use, NYHA class $>3$, serum creatinine, haemoglobin, diabetes mellitus and an average mean AV gradient to be predictive for TAVI futility. ${ }^{21}$ This was defined as 1-year composite of mortality, stroke, lack of functional-class improvement (by NYHA class) and readmissions ( $\geq 1$ month after the procedure). They report a futility rate of $15 \%$, much lower than the futility rate we report, probably because they also included procedural parameters, and furthermore only included uncomplicated, optimal TAVI procedures, all with device success and without any major and/or debilitating complications.

The definition of a futile TAVI is varying in literature and is, and in our opinion should be, under debate. The definition implies that there was no objective measurable benefit for the patient and the procedure should not have been performed. The same applies for residual impairment as a surrogate for TAVI futility. In both cases, there might still be patient benefit by any decrease in dyspnoea/ angina or other more underexposed AS-related symptoms such as fatigue or general unwellness. Furthermore, TAVI removes a mortality risk of untreated aortic valve stenosis, which may also pivotally reduce anxiety and improve patient confidence. From the patients' perspective, this could be one of the most important outcome measures since many TAVI patients might probably value the quality of the remaining lifespan greater than the actual length of it. In the near future, less-strict criteria for TAVI futility, that is, using a shorter life expectancy and incorporating patients' perceived outcomes, could be used. This further defines futility into an objective, real-life measure of the absence of actual benefit. In our experience, patients with a treated AS have a higher exercise tolerance and are more capable and confident to undertake physical activity and live a more independent life. This might have been attributing to the positive patient-reported outcomes of the TAVI treatment in this study. Incorporating PROMs into a new TAVI futility definition would lower the amount of actual 'futile' TAVI procedures. Furthermore, the current warranted 1-year life expectancy threshold is merely based on expert opinion. Since major complications after TAVI are decreasing strongly, as do the costs, this threshold could be debated and become more patient centred. Quality of life (QoL) assessment was not reported in this study and should be an additional target for TAVI. A wide range of QoL questionnaires were developed, but many of them are difficult to assess in clinical practice in the very elderly with symptomatic AS and comorbidities.

\section{Patient-reported outcomes and value-based healthcare}

In this study, we contacted all accessible patients and included patient-reported outcomes in our follow-up, where other (cohort) studies merely focus on technical procedural outcomes and mortality. This gave us the unique opportunity to discuss value-based healthcare concerning TAVI treatment and gave valuable insight in the patient-perceived effects of TAVI. We reported a large amount of patients who would undergo TAVI once again in retrospection $(89.9 \%$ of patients reached), even in the patients of who we judge to have no clear benefit (ie, no NYHA-class decrease). This is probably originating in the minimal invasive character of the TAVI, combined 
with the positive effects on self-confidence. These results make TAVI a very valuable treatment, adjudicated from a viewpoint of value-based healthcare, where they may have been designated as futile by the current guidelines. When considering benefits, one should focus on clinical endpoints and rather also include patient-perceived values of the procedure. In this study, we have a lost-tofollow-up of $12 \%$ for PROMs in patients who were alive at the moment of telephone follow-up. Corresponding with the aforementioned predictors for mortality, the baseline characteristics of the telephone follow-up cohort differed slightly from the total population with less comorbidities. One should keep in mind that this could give a bias to the results as this might be due to a bad outcome after TAVI. Nevertheless, this still accounts for a small proportion of patients. For further research, it would be useful to create a prediction model based on large prospective studies for which we identified possible predictors in this hypothesis-generating study, and incorporate patient-reported outcomes in the definition of futility of TAVI treatment.

\section{Limitations}

The present analysis was conducted on a single-centre non-randomised cohort and has therefore inherent limitations to such design, including the described part of missing values regarding the follow-up of functional status, and at the start of the cohort, clinical frailty assessment was not yet common. However, it still answers clinically relevant questions. Moreover, NYHA class as a functional outcome is not completely objective and may not reflect a patient's improvement in daily functioning, possibly explaining the discrepancy between the PROMs and the presence or absence of functional improvement we report. However, the NYHA scale is generally used in clinical follow-up, so it is the closest functional parameter to generalise to daily clinical practice. Furthermore, survivorship bias and placebo effect could have substantially biased the positive PROMs we report, which one should take into account when assessing the patient-reported value of the TAVI treatment.

In this study, we did not report about patients who were denied for a procedure and can therefore not analyse the effects of optimal medical therapy versus a TAVI procedure. This was, however, already demonstrated in the PARTNER I trial. ${ }^{22}$

\section{CONCLUSION}

Lower albumin and non-transfemoral access route were predictors for guideline-defined TAVI futility, defined as mortality within 1 year or no objective symptomatic improvement in NYHA class. Futility according to this definition occurred frequently in this study, contrasting to much more positive patient-reported outcomes. The majority of patients would undergo a TAVI again, underlining the patients' experienced value of TAVI and putting the definition of TAVI futility further on debate. In the near future, less-strict criteria for TAVI futility, that is, using a shorter warranted life expectancy and incorporating patients' perceived outcomes, may be desirable.

Contributors MSVM, JV, MA, JB and MMV conceived and designed the study. MSvM, JV and FvK acquired the data. MSvM and JV did the statistical analysis. MSvM and JV drafted the manuscript. FvK, MA, JPSH, AHGD, JJW, RJdW, JJP, JGT, KTK, JB and MMV contributed to the interpretation of the results and critical review of the manuscript. All authors have read and approved the manuscript. All authors have also sufficiently contributed to conception and design of the study, analysis and interpretation of data, drafting of the manuscript or revising it to justify authorship. Guarantors of the study: MSvM, JV.

Funding The authors have not declared a specific grant for this research from any funding agency in the public, commercial or not-for-profit sectors.

Competing interests JB receives an unrestricted research grant from Edwards Lifesciences and is proctor for Edwards Lifesciences.

Patient consent Not required.

Ethics approval AMC METC supplied a waiver.

Provenance and peer review Not commissioned; internally peer reviewed.

Data sharing statement Questions regarding data sharing can be addressed to the corresponding author.

Open access This is an open access article distributed in accordance with the Creative Commons Attribution Non Commercial (CC BY-NC 4.0) license, which permits others to distribute, remix, adapt, build upon this work non-commercially, and license their derivative works on different terms, provided the original work is properly cited, appropriate credit is given, any changes made indicated, and the use is non-commercial. See: http://creativecommons.org/licenses/by-nc/4.0/

\section{REFERENCES}

1. Baumgartner H, Falk V, ESC Scientific Document Group. 2017 ESC/ EACTS guidelines for the management of valvular heart disease. Eur Heart J 2017;38:2739-91.

2. Nishimura RA, Otto CM, Bonow RO, et al. 2017 AHA/ACC focused update of the 2014 AHA/ACC guideline for the management of patients with valvular heart disease: a report of the American College of Cardiology/American Heart Association Task Force on Clinical Practice Guidelines. J Am Coll Cardiol 2017;70:252-89.

3. Biancari F, Juvonen T, Onorati F, et al. Meta-analysis on the performance of the EuroSCORE II and the Society of Thoracic Surgeons Scores in patients undergoing aortic valve replacement. $J$ Cardiothorac Vasc Anesth 2014;28:1533-9.

4. Martin GP, Sperrin M, Ludman PF, et al. Inadequacy of existing clinical prediction models for predicting mortality after transcatheter aortic valve implantation. Am Heart $J$ 2017;184:97-105.

5. Reardon MJ, Van Mieghem NM, SURTAVI Investigators. Surgical or transcatheter aortic-valve replacement in intermediate-risk patients. N Engl J Med 2017;376:1321-31.

6. Leon MB, Smith CR, PARTNER 2 Investigators. Transcatheter or surgical aortic-valve replacement in intermediate-risk patients. $N$ Engl J Med 2016;374:1609-20.

7. Puri R, lung B, Cohen DJ, et al. TAVI or no TAVI: identifying patients unlikely to benefit from transcatheter aortic valve implantation. Eur Heart J 2016;37:2217-25.

8. Schneiderman LJ, Jecker NS, Jonsen AR. Medical futility: its meaning and ethical implications. Ann Intern Med 1990;112:949-54.

9. Liao YB, He ZX, Zhao ZG, et al. The relationship between chronic obstructive pulmonary disease and transcatheter aortic valve implantation-a systematic review and meta-analysis. Catheter Cardiovasc Interv 2016;87(Suppl 1):570-8.

10. Chopard R, Meneveau N, Chocron S, et al. Impact of chronic obstructive pulmonary disease on Valve Academic Research Consortium-defined outcomes after transcatheter aortic valve implantation (from the FRANCE 2 Registry). Am J Cardiol 2014;113:1543-9.

11. Conrotto F, Salizzoni S, Andreis A, et al. Transcatheter aortic valve implantation in patients with advanced chronic kidney disease. Am J Cardiol 2017;119:1438-42.

12. Ferro CJ, UK TAVI Steering GroupNational Institute for Cardiovascular Outcomes Research. Impact of renal function on survival after transcatheter aortic valve implantation (TAVI): an analysis of the UK TAVI registry. Heart 2015;101:546-52. 
13. Arnold SV, Afilalo J, U.S. CoreValve Investigators. Prediction of poor outcome after transcatheter aortic valve replacement. J Am Coll Cardiol 2016;68:1868-77.

14. Arnold SV, Reynolds MR, PARTNER Investigators. Predictors of poor outcomes after transcatheter aortic valve replacement: results from the PARTNER (Placement of Aortic Transcatheter Valve) trial. Circulation 2014:129:2682-90.

15. Ribeiro HB, Rodés-Cabau J. The multiparametric FRANCE-2 risk score: one step further in improving the clinical decisionmaking process in transcatheter aortic valve implantation. Heart 2014;100:993-5.

16. Porter ME. A strategy for health care reform - toward a value-based system. N Engl J Med 2009;361:109-12.

17. Kappetein AP, Head SJ, Valve Academic Research Consortium (VARC)-2. Updated standardized endpoint definitions for transcatheter aortic valve implantation: the Valve Academic Research Consortium-2 consensus document (VARC-2). Eur J Cardiothorac Surg 2012;42:S45-60.
18. Otto CM, Kumbhani DJ, Alexander KP, et al. 2017 ACC Expert consensus decision pathway for transcatheter aortic valve replacement in the management of adults with aortic stenosis: a report of the American College of Cardiology Task Force on Clinical Expert Consensus Documents. J Am Coll Cardiol 2017;69:1313-46.

19. Martin GP, Sperrin M, Ludman PF, et al. Novel United Kingdom prognostic model for 30-day mortality following transcatheter aortic valve implantation. Heart 2018;104:1109-1116.

20. Abdelghani M, Cavalcante R, Miyazaki Y, et al. Prevalence, predictors, and prognostic implications of residual impairment of functional capacity after transcatheter aortic valve implantation. Clin Res Cardiol 2017;106:752-9.

21. Zusman O, Kornowski R, Witberg G, et al. Transcatheter aortic valve implantation futility risk model development and validation among treated patients with aortic stenosis. Am J Cardiol 2017;120:2241-6.

22. Leon MB, Smith CR, PARTNER Trial Investigators. Transcatheter aortic-valve implantation for aortic stenosis in patients who cannot undergo surgery. N Engl J Med 2010;363:1597-607. 